https:/ / creativecommons.org/ licenses/ by/ 4.0/

\title{
IMPORTANCIA DEL TALENTO HUMANO Y HERRAMIENTAS TECNOLÓGICAS EN EL DESARROLLO ORGANIZACIONAL, PARA LA MEJORA DE LA PRODUCTIVIDAD LABORAL
}

\section{Importance of human talent and technology tools in organizational development, for the improvement of labor productivity}

ANA MARÍA MARTíNEZ URUEÑA ${ }^{1}$, MARIA ALEJANDRA MATEUS MORENO ${ }^{2}$

Recibido:07 de junio de 2020. A ceptado:20 de junio de 2020

DOI: http://dx.doi.org/10.21017/rimci.2020.v7.n14.a88

\begin{abstract}
Resumen
El desarrollo organizacional permite la eficiencia y eficacia de las empresas, la clave para qué este proceso sea exitoso está en la participación de las personas que conforman la organización, sin importar su rol y el cargo que desempeñe. El resultado de un adecuado desarrollo organizacional tendrá un avance considerable en cuanto se genere la motivación, la creatividad, la productividad, el sentido de pertenencia en sus colaboradores, logrando el uso adecuado del capital humano y de las herramientas tecnológicas, lo que contribuirá a generar ventajas competitivas que permita el aumento de los beneficios de to da la organización.
\end{abstract}

Palabras clave. G estión organizacional; producción; estructura social; calidad y bienestar laboral; tecnologías de la información.

\begin{abstract}
O rganizational development allows the efficiency and effectiveness of the companies, the key for this process to be successful is in the active participation of the people who make up the organization, regardless of their role and the position in which they perform. The result of an adequate organizational development will largely be in how motivation, creativity, productivity and a sense of belonging are generated in the collaborators, in order to achieve the proper use of human capital and technological tools, which will contribute to the creation of competitive advantages that allow the increase of the benefits of the whole organization.
\end{abstract}

Keywords. O rganizational management, production, social structure, quality and labor welfare, information techno logies.

\section{INTRODUCCIÓN}

Los cambios rápidos dentro del entorno organizacional han exigido procesos y estructuras organizacionales quesean mucho másflexibles y que brinden mayor capacidad de respuesta que las estructuras tradicionales, pero para esto, se requieren nuevas capacidades individual es y empresariales que puedan impulsar a los trabajadores asumir riesgos en la organización. El Desarrollo Organizacional busca lograr un cambio planeado dela organización conformeen primer término a las necesidades, exigencias o demandas de esta [1].

1 Estudiante de Ingeniería Industrial, Semillero de Investigación, Grupo O CA. Corporación U niversitaria Republicana. Correo electrónico: am.uruena@ urepublicana.edu.co

2 Estudiante de Ingeniería Industrial, Semillero de Investigación, Grupo OCA. Corporación Universitaria Republicana. Correo electrónico: ma.mateus@urepublicana.edu.co 
El desarrollo organizacional es el diseño de procesos, estructura, base y fundamento para construir ideas, esquemas o el progreso de una determinada empresa u organización, estas organizaciones generalmente deben estar conformadas por personas que tengan un conjunto de diferente valores, estilos y habilidades; tecnologías con distintas características; procesos y estructuras que reflejan diversos tipos de relaciones entre el entorno y la organización.

El desarrollo organizacional seencuentra conectado con la calidad de vida en el trabajo y la productividad, según[2], en su libro "Fuera de la crisis", plantea la visión de cómo las organizaciones pueden asegurar la calidad de sus productos a partir de la importancia que tiene su personal al momento de la generación de bienes y/ o servicios, planteando además, el esquema de mejora permanente derivado de una serie de pasos como son planeación, ejecución, verificación y estandarización de procesos, esto, a partir de la perspectiva de calidad total que aporta elementos fundamentales en el análisis de contextos empresariales específicos, permitiendo establecer los niveles de productividad esperados por cada uno de los trabajadores, teniendo en cuenta, el espacio laboral, Ios tiempos asignados, y las características asociadas a la labor, en conjunto con las características de los colaboradores en términos de capacidades[3].

La Productividad Laboral y su impacto en el desempeño empresarial, son estudiados desde

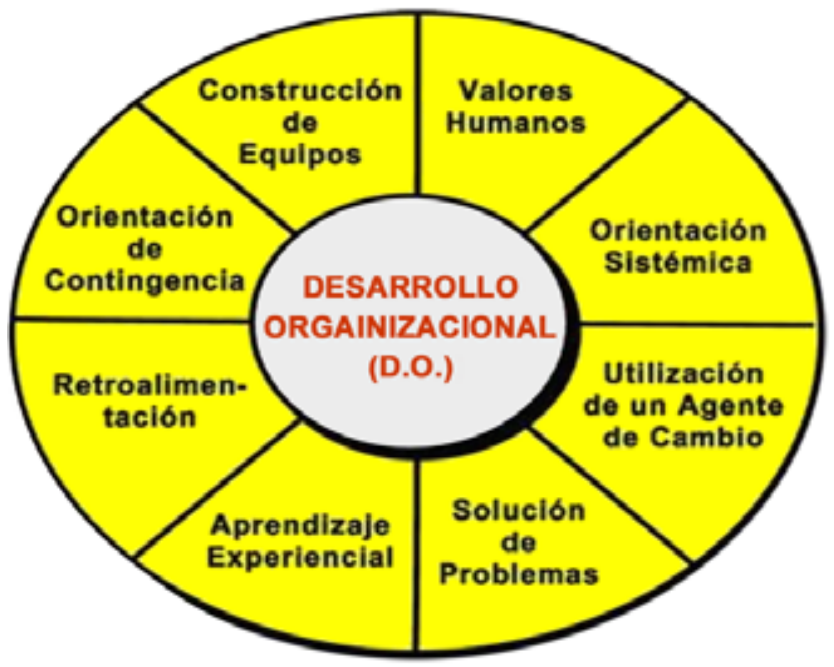

Fig. 1. Desarrollo Organizacional Ambiental[4]. Fuente: diferentes enfoques y disciplinas [5]. Desde la ingeniería, se concibe como el resultado del sistema productivo generado por el desempeño delas personas en un lugar de trabajo y está asociado con la optimización de los recursos materiales, financieros y tecnológicos de la organización [6].

La productividad se considera como la variable objetiva para la medición del progreso competitivo de una organización, indica como la entidad gestiona los recursos disponibles en pro de un objetivo, esta administración evoluciona gracias a las realizaciones y al desempeño del factor humano. Para evaluar la productividad laboral que se divide en estos 4 principales factores:

- Capital físico, es la cantidad de equipos y estructuras queseutilizan para producir bienes y servicios.

- Capital humano, son los conocimientosy calificaciones que adquieren los trabajadores por medio dela educación, la formación y la experiencia.

- Recursos naturales, son los factores que intervienen en la producción de bienes y servicios y que son aportados por la naturaleza

- Conocimientos tecnológicos, son lacomprensión dela sociedad sobrelas mejores formas de producir bienes y servicios [7].

El estudio de la productividad laboral desde estos 4 factores se puede determinar la necesidad en quelos empresarios deben saber la importancia de capacitar al personal, promover y facilitar su participación en la mejora del proceso productivo y a su vez el análisis eimplementación de tecnologías de la información, como variables de la gestión del desarrollo organizacional.

La clave del desarrollo en las organizaciones está en tener en cuenta la creatividad, motivación, compromiso de las personas que participan en ella fundamentado en que los altos niveles de competitividad exigen nuevas formas decompromiso, de ver los hechos, de decidir y dirigir, de pensar y sentir, así como de gestionar las relaciones humanas en las organizaciones de una forma más efectiva. La G estión del desarrollo organizacional, el capital humano es definido como la mano de 
obra dentro de una empresa, es el recurso más importante y básico, ya que es el que desarrolla el trabajo de la productividad de bienes o servicios con la finalidad de satisfacer necesidades y obtener una utilidad [8]. La tecnología y estructura organizacional se centra en la trasformación del conocimiento convirtiéndolos en elementosfuncionales para el desarrollo de las actividades, los métodos utilizados para que la trasformación funcione, es el análisis de los colaboradores participantes, el conocimiento de materiales e instrumentos y por último el control que le damos al desarrollo de estas actividades.

El desarrollo organizacional se enfoca esencialmente en el lado humano dela empresa, es decir, los valores, las actitudes, las relaciones, el clima y la cultura organizacional, este desarrollo busca dirigirse más sobre las personas que sobre los objetivos económicos, la estructura o las técnicas que emplea la organización. El mundo se mueve y transforma a velocidades cada vez más rápidas dados los niveles competitivos de las organizaciones [9], el talento humano en el desarrollo organizacional es partefundamental del desarroIlo de la productividad empresarial, está enfocado en el desarrollo del capital humano, a mayor identificación y compromiso con la empresa para la cual selabore, mayor eficacia se tendrá en el desarroIlo de las funciones y esto beneficiara la economía y la sociedad, no solo a la empresa sino al país. Es importante que la organización tenga en cuenta que las personas son un factor crítico y definitivo en el desarrollo de las organizaciones. Para potenciar este campo se han formado las áreas de gestión humana que a través de sus programas y procesos se encaminan a lograr estefin. Estos procesos buscan comprender al ser humano en su totalidad, dotándolo de herramientas que permiten una adaptación al mundo organizacional, pero también con conocimientos y habilidades que mejoran sus vidas, la de sus familias y la sociedad. La perspectiva es brindar beneficios prestacionales a los colaboradores, no sólo en el marco teórico de la paga por un servicio, sino buscar salarios emocionales por los cuáles los trabajadores se sientan identificados, satisfechos, comprometidos y lo más importante hagan parte de un equipo que les garantice el desarrollo y crecimiento, no solo económico, sino que los proyectey les asegure una mejor calidad de vida para ellos y su familia.

\section{IMPORTANCIA DEL DESARROLLO ORGANZACIONAL}

El desarrollo organizacional ha presentado evoluciones de gran significado a través de la historia; un concepto que tiene todos los enfoques es el realizado por Allairey Firsirotu donderefiere “Es un compuesto de variables endógenas y factores exógenos", tales como:

- Un sistema socio estructural compuesto de relaciones entre las estructuras, las estrategias, las políticas y otros procesos formales de gerencia.

- Un sistema cultural que agrupa los aspectos expresivos y efectivos de la organización en un sistema colectivo de símbolos significativos.

- Los empleados como individuos, con su talento, sus experiencias y su personalidad, creadores, formadores unificadores de importancia, queelaboran activamente una realidad organizacional coherente a partir del flujo continuo de acontecimientos [10].

En las organizaciones como sistemas sociales, sin lugar a duda uno de los componentes clave es el factor humano, con su comportamiento competente, dado por la contribución que efectúan las personas a la organización a favor de los objetivos dela institución y la sociedad.

Para afrontar la demanda de institucionalizar I os cambios, la gerencia moderna ha creado estrategias de desarrollo organizacional, que permiten a la alta gerencia con participación de los miembros en las empresas planificar y afianzar las innovaciones requeridas, especialmenteen los ámbitos sociológicos y metodológicos. Estos cambios implican una nueva cultura organizacional y pueden ser operados desde distintos niveles. A Igunos autores se refieren a los niveles: estructural, tecnológico y del comportamiento.

La importancia que se le da al desarrollo organizacional deriva deque el recurso humano es decisivo para el éxito o fracaso decualquier organización. En consecuencia, su manejo es clave para el éxito empresarial y organizacional en general, comenzando por adecuar la estructura de la organi- 
zación (organigrama), siguiendo por una eficiente conducción de los grupos de trabajo (equipos y liderazgo) y desarrollando relaciones humanas que permitan prevenir los conflictos y resolverlos rápiday oportunamentecuando setenga indicios desu eclosión [11]. El desarrollo organizacional aborda problemas de comunicación, de dirección, conflictos entre grupos, temas de identificación y destino de la institución, satisfacción y motivación de los miembros de la organización, así como cuestiones de mejora institucional, por lo que se considera un instrumento por excelencia para la gestión del cambio en busca del logro de una mayor eficiencia organizacional y productividad.

La productividad está conectada de forma directa a la cultura organizacional de las empresas, los resultados de una organización, el proceso de dirección es un elemento fundamental, ya que es aquí donde se movilizan los recursos humanos, materiales, financieros einstalaciones para prestar servicios a una población determinada. Los modelos económicos de las ciudades que se determina como el conjunto unificado de ideas presunciones básicamente inventadas a través del tiempo y las revoluciones fundamentales, las cuales modificaron los conceptos principales del marco social una variable estratégica en la evolución de los factores fundamentales es decir en el desarrollo económico, modelo empresarial, competencias humanas entre otras.

Partiendo de varios conceptos y definiciones Allaire y Firsirotu inyectan a la definición de organización un contexto fundamental para el desarrollo de la investigación, al citar un sistema socio estructural compuesto de relaciones entre las estructuras fundamental mente se inicia analizando el contexto estado-empleador-trabajador-maquinaria. Las estrategias serían las formas utilizadas de manera individual que garantizan el beneficio colectivo, con las políticas serefiereala fundamentación jurídica deberes y derechos, conjunto de leyes que brindan un ordenamiento a nivel laboral, incluyendo la representación individual del líder de manera gerencial, puesto que hay una gran diferencia en relación laboral y relación de trabajo.

Las variables de esta definición serian el sistema cultural y los empleados como individuos incluye el bienestar organizacional, beneficios, prestaciones, es decir el desarrollo organizacional que estudia al individuo con el propósito de aplicar estos conocimientos en las mejoras de la organización según los planteamientos de Gibson, Ivancevich, Donelly y Konospake "Campo de estudio que se sustenta en la teoría, método y principios dediversas disciplinas para aprender acerca delas percepciones, valores, capacidades de aprendizaje de los individuos mientras trabajan en grupo y dentro ella, para analizar el efecto del ambientedela organización los recursos humanos, misiones, objetivos y estrategias" [12].

Para Chiavenato “El comportamiento organizacional retrata la continua interacción y la influencia recíproca entre las personas y las organizaciones", estemarco o concepto organizacional inicia sus pinares en el año 1962 cuando se crea una teoría con la idea hombre, organización y ambiente, es decir se convierte en un proceso sistemático y planificado, en que se utilizan los principios de la ciencia del comportamiento para mejorar la efectividad individual en un proceso organizacional.

A la hora de desarrollar los procesos de gestión del capital humano en las organizaciones hay quetener presenteque existen procesos en los cuales intervienen todos los recursos de la organización como son: el capital financiero, la producción científica, las tecnologías, el tiempo y las personas propiamentedichas; dondeel uso adecuado deuna comunicación efectiva y eficienteinfluyedeforma positiva en el logro de los objetivos de trabajo. De ahí la importancia del capital humano en el comportamiento organizacional, ya que el estudio y aplicación de los conocimientos sobre la manera en la que las personas, tanto individual como en grupos, actúan en las organizaciones favorece el desarrollo de estas [12].

\section{Características del desarRollo ORGANIZACIONAL}

El desarrollo organizacional, en sus principios teóricos aporta un conjunto de ideas acerca del hombre, la organización y el ambiente; orientadas a propiciar el desarrollo y crecimiento de sus potencialidades representadas en: competencias, habilidades y destrezas.

El desarrollo organizacional busca perpetuarse en el pensamiento administrativo al presentar

Rev. Ingeniería, M atemáticas y Ciencias de la Información Vol. 7 / N úm. 14 / julio - diciembre de 2020; pág. 117-126 
desde sus orígenes, un concepto dinámico de las organizaciones, dela cultura y el cambio organizacionales. Conceptualmente está conformado por dos descriptores relevantes: Desarrollo y Organización y éstos desdeel punto de vista administrativo podemos definirlos como:

D esarrollo: la palabra implica una acción y efecto de desarrollar o desarrollarse; es decir, aumentar, acrecentar, perfeccionar las actividades que realiza una persona o una organización. Desdela connotación administrativa, significa la implementación de estrategias para la formulación de un proceso de cambio planeado, que resulta lento y gradual que conduce al exacto conocimiento de las fortalezas, debilidades de la organización y al aprovechamiento delas oportunidades que coadyuvan la plena realización de sus potencialidades[13].

O rganización: es una acción de organizar, disponer o establecer de una forma de regular; bajo el enfoque de las ciencias administrativas: es la coordinación de las actividades de todos Ios individuos queintegran una empresa con el propósito deobtener el máximo aprovechamiento posiblede elementos materiales, técnicos y humanos, en la realización de los fines, metas y objetivos que la propia empresa persigue [14].

La conjunción de ambos conceptos nos aporta las primeras directrices teóricas, al incluir el concepto de cambio. El cambio, debe orientarse a: redefinir creencias, actitudes, valores, estrategias y prácticas para que la organización pueda adaptarse mejor a las modificaciones del entorno y evolución defenómenos administrativos imperantes en el medio.

W.G. Bennis, uno delos principales iniciadores deesta actividad, enuncia lo siguiente “Desarrollo Organizacional es una respuesta de cambio, una compleja estrategia educativa cuya finalidad es cambiar las creencias, actitudes, valores y estructuras de las organizaciones, en tal forma que estas puedan adaptarse mejor a nuevas tecnologías, mercados y retos, así como al ritmo vertiginoso del cambio", de esta definición se toman las siguientes características:

\section{D ebe ser:}

- Proceso dinámico, dialectico y continuo.
- Decambios planeados a partir dediagnósticos realistas de situaciones concretas.

- Renovando los sistemas abiertos y económicos del comportamiento empresarial.

\section{Requiere}

- Visión global.

- Enfoque de sistemas abiertos.

- Direccionamiento.

- Sistematización de procesos.

- Desarrollo decompetencias colectivas, individuales, de sistemas y subsistemas.

\section{Implica}

- Valores Humanísticos.

- A daptación al cambio social.

- Análisis y modificación de hábitos y comportamientos.

\section{IV . ESTRATEGIA ORGANIZACIONAL}

Los constantes cambios en los que se ven envueltas las organizacionesy su necesidad deadaptación a un entorno impredecible para mantener su competitividad, exigen nuevas formas de organizar el trabajo [15].

Se considera al fundamento táctico o la arquitectura de la parte gerencial, según la proyección y el pensamiento se enfocarían en alguno de los siguientes 4 grupos:

- A utocrático: Depende del poder, gerente con orientación de autoridad en consecuencia a este modelo de comportamiento organizacional el trabajador se caracteriza por su obediencia y dependencia. En este enfoque el gerente tendrá como resultado del desempeño al go mínimo y en consecuencia un costo elevado en el aspecto humano.

- Custodia: En esteenfoqueel colaborador depende de la organización, esta ofrece garantías legales. El modelo depende delos recursos económicos y se orienta a la satisfacción de necesidades de seguridad del trabajador, el gerente se orienta al dinero en sí y la dependencia al trabajador es a la empresa. 
- De apoyo: En este modelo no importa el dinero y el poder sino el apoyo del líder hacia los empleados, el gerente se orienta al apoyo de sus trabajadores, los resultados que se obtienen son de gran participación por parte del trabajador y un desempeño caracterizado por impulsos.

- Colegial: Este es basado en una sensación de compañerismo entre los empleados, este se caracteriza por la dependencia de la sociedad, el gerente seorienta a una participación en equipo y el empleado tiene un gran sentido deresponsabilidad y autodisciplina.

Dependiendo el modelo utilizado hay un resultado favorable o desfavorable en cuanto a términos económicos y productivos de una empresa o de un país.

En un panorama económico a nivel global la capitalización deun paíssi tienevariaciones según los medios de producción utilizados, la implementación demodelos empresariales, con políticas claras organizadas, los países con más rentabilidad son aquellos en los quese encuentra una mejor gestión organizacional es decir un marco de desarrollo industrial en los que el empleado se siente parte del equipo, no como forma de explotación, siente que reconocen sus habilidades y competencias.

Hablado en un panorama económico, los países con las estructuras fortalecidas a nivel del desarrollo organización, con un enfoque primario en programas que sustenten un manejo empresarial con objetivos específicos, claros, concretos y colectivos pero encaminados a una meta el progreso, en macroeconomía serian aquellos queexpresan el valor monetario de la producción de bienes y servicios de una forma más productiva y eficiente, estas economías están reguladas por altos niveles tecnológicosy al tos niveles de satisfacción en cuanto a salarios, horarios y calidad de vida.

\section{LA TECNOLOGÍA DE LA INFORMACION EN EL DESARROLLO ORGANIZACIONAL}

Lastecnologías dela información inician su auge a nivel mundial, con el propósito fundamental de dar avances a nivel social y económico, se utiliza en los negocios a partir de los años 70 se incorpo- ran en el manejo de procesadores, equipos, manejo de datos y sistemas de información, su objetivo esla gestión denegocio pequeñas mediana y grades empresas. En Colombia, son reguladas por el Ministerio de las TIC, el Ministerio de Comunicaciones tuvo cambio importante en el año de 1976 donde se reestructura y adecua la planta con acuerdo a las exigencias dela década, en el año 2009 ya como tal se convirtió en el Ministerio de Tecnologías de la información (TIC) fortaleciendo la protección y seguridad de los usuarios.

Las TICS se adiciona como valor agregado a los procesos de las compañías, permitiendo el intercambio de información y un modelo más organizado en cuanto a todo el esquema operativo, funcionan en todas las dependencias de una compañía, y seencuentran como partefundamental del mapa de procesos en una organización, su objetivo es la gestión empresarial.

La capacidad tecnológica se ha evaluado teniendo en cuenta factores como visión y estrategia deTI, conocimiento, activos einfraestructura dehardware y software. Sereconoceel carácter multidimensional del concepto de capacidad tecnológica como el conjunto de elementos de infraestructura de TI, arquitectura de $\mathrm{TI}$, recurso humano y relaciones basadas en TI No es, entonces, la medición de capacidad tecnológica un asunto trivial de inventario de hardware y software, por cuanto el mejoramiento del negocio a través de la tecnología, no se da por la tecnología en sí misma, sino por los cambios e innovaciones que del uso de ella se derivan. Aunque los recursos tecnológicos pueden conseguirse con relativa facilidad, deben estar acompañados de unas habilidades y capacidades en la organización queconlleven a su aprovechamiento [16].

\section{HeRramientas digitales GESTIÓN EMPRESARIAL}

Pero centremos el análisis en la Cuarta Revolución Industrial. La adopción masiva de las tecnologías digitales originadas en la última década del siglo XX, como Internet y los teléfonos móviles inteligentes, junto con el crecimiento extraordinario dela computación y la capacidad dealmacenamiento a un costo menor, así como otras tecnologías digitales exponenciales, están transformando radicalmente el mundo, cambiando profundamente 
los 10 patrones de las relaciones personales, las organizaciones empresariales y, en general, la forma en que se crea el valor económico [17].

La experiencia con el desarrollo dela automatización y los robots durantelos últimostreinta años parece que no es del todo concluyente. En efecto, como hemos visto, si bien no crece el desempleo a nivel agregado, sí tenemos un mercado laboral más polarizado. Pero es quizás demasiado aventurado extrapolar el comportamiento del empleo en los últimos años hacia el futuro [17].

Sin embargo, nadie puede poner en duda que los robots, el Internet de las Cosas, Ia Inteligencia Artificial y el Big Data irán desplazando a los trabajadores actuales deal gunas actividades, mientras que se desarrollarán otras nuevas que darán lugar ala creación denuevosempleos [16]. Teniendo como complemento los siguientes conceptos y el enfoque que se le ha dado a la Tecnología - Empleado:

- Redes sociales: Las redes sociales nacieron desdeel siglo XVIII y en el siglo XIX seutilizó el término de redes sociales y a partir de estos momentos las universidades empezaron a investigar sobre el impacto en las organizaciones, el segundo auge de este término fue la extensión de tecnologías de comunicación con el crecimiento del internet y el desarrollo de nuevo software que condujo a la creación de redes sociales las cuales integraron el mundo físico con el mundo virtual teniendo un mayor crecimiento en el año 2002 y hasta la fecha.

Seha convertido en una herramienta fundamental para las organizaciones para el funcionamiento operacional construyendo nuevos canales de comunicación tanto con los clientes externos como los internos, al igual se ha convertido en una herramienta para su crecimiento y expansión comercial, generando posicionamiento eimagen consiguiendo más clientes a través de amigos, seguidores interconectados entre sí.

A Igunas de las redes sociales que se utilizan las organizaciones son: Facebook, Instragram, Twitter, Google, Youtube, Linkedln y Whatsapp.

- Acceso remoto y movilidad: Encontrar el talento humano y retenerlo es difícil, por lo tanto, las empresas han implementado el trabajo desde casa disminuyendo el costo y aumentando la productividad delos colaboradores, rompiendo también barreras geográficas obteniendo un equipo más diverso y multicultural, con habilidadesy clientes de todo el mundo. La cultura remota es saludable y sólida produciendo trabajadores felices y productivos.

- Computación en nube: Es un beneficio que tienemuchas más ventajas para las empresas colombianas, ya que se trata de almacenamiento y uso de información por medio de servidoresconectadosal internetreemplazando las máquinas locales con infraestructura virtual, existen 3 formas de uso de la nube, pública que es para todos los clientes, privada que es cuando se les otorga ciertos permisos a las personas para el ingreso, y las dos quees una combinación parael servicio a ofrecer. Es eficientey de bajo costo aumentado la productividad y fácil deexpansión, debemos también tener en cuenta que también sirve para los trabajadores que se encuentren en diferente ciudad o país se puedan conectar sin inconvenientes, asistir a conferencia o validar archivos haciendo que no haya ninguna dificultad para las empresas ni trabajadores en su eficiencia y productividad empresarial.

- Big data: Es una matriz que no tiene límites para la recopilación de información el cual tienecomo funcionamiento la administración y análisis de bases datos hay que tener en cuenta queel almacenamiento deinformación por cada 2 años se duplica por consecuencia de la revolución digital, con algoritmos e interfaces para la sistematización de una página o software, siendo más eficiente para las empresas, generando menor riesgos es sus procesos, disminuyendo el costo, más rápido la toma de decisiones y en generar nuevos productos y servicios.

- Internet de las cosas: Es la interconexión digital de objetos cotidianos con el internet, cosas con las que convivimos diario y antes no estaban conectadas a la red, en las empresas vemos esto más centrado en los clientes, ya quesi al cliente le guste o tenga más ideas de tecnología las empresas tendrán mayores 
ventas y mejora en sus productos, también tener claro que en el presente no contamos con mucha tecnología de punta, pero podemos llegar a alcanzar el uso de casas y electrodomésticos con inteligencia artificial o vanguardistas para nuestra época y clientes tecnológicos dándolevalor agregado a el país y a nuestras empresas.

El modelo gestión se enmarcaría según lo analizado anteriormentecomo tecnología empresarial que ha tenido un cambio considerable frente a los usos y la seguridad informática, convirtiéndoseen un factor indispensable e importante para la comunicación empresarial, cuando las empresas introducen las tecnologías en sus procesos y direccionamiento, se vuelven más competitivas y vanguardista en los servicios, los productos prestados, teniendo una mayor rentabilidad y avances frente a los procesos tanto como al que adquiere los servicios es decir el cliente.

Por otra parte la tecnología organizacional y a su vez la vanguardia se centra en la trasformación del conocimiento convirtiéndolos en elementosfuncionales para el desarrollo de las actividades, los métodos utilizados para que dicha trasformación funcione, es el análisis delos colaboradores participantes, el conocimiento de materiales e instrumentosy por último el control queledamosal desarrollo de estas actividades; la Cuarta Revolución Industrial supondrá un cambio de paradigma en el mercado laboral, con un impacto directo en el empleo, pero su efecto no sólo se circunscribirá a esteámbito, sino que afectará directamente al fundamento del Estado de Bienestar de la sociedad actual [17]. A plicando la tecnología se tiene quetener claridad de la siguiente dimensión, la regularización quees darle forma a los procesos y a los procedimientos de tal manera que sean eficaces y reglamentarios. Cabe destacar que entre la organización sea más grande va a hacer menos difícil la estructura de la tecnología y en el caso contrario delas organizaciones pequeñas tendrá un impacto frente a la estructura y la productividad.

\section{Talento humano, una mejora A LA PRODUCTIVIDAD}

Levi y Anderson definen la calidad de vida laboral como el conjunto formado por el bienestar fí- sico, mental y social, del modo como es percibido por cada sujeto [18]. La calidad de vida de un trabajador en una organización o empresa independiente cual sea su rol a desempeñar, no solamente depende desu estado deánimo sino de su satisfacción al bienestar que le genera dicha estructura. El bienestar del trabajador implica tener buenas relaciones personales en su entorno laboral y familiar, tener buena salud, acceso a bienes materiales y beneficios prestacionales, para así llevar una vida digna.

\section{"La O M S define un entorno de trabajo saluda- ble como aquel en el que los trabajadores y di- rectivos colaboran en utilizar un proceso demejora continua para proteger y promover la salud, segu- ridad y bienestar de los trabajadores" [18].}

Normativamente en Colombia el trabajo está regulado y definido en la constitución nacional y en el código laboral "Ley núm. 50 de $\mathbf{2 8}$ de diciembre de 1990, por la que se introducen reformas al Código Sustantivo de Trabajo y se dictan otras disposiciones", [19] trabajo es igual a derecho en condiciones dignas y justas el estado es el cargado de garantizar, la equidad, la igualdad y las condiciones al trabajador, y el trabajador es el quejuega el papel más importanteen cuanto a producción se habla puesto que de la energía de este se despliega el bien o servicio brindado.

Inicial mentelas empresas buscaban queel trabajador solo se dedicara a producir sin importar las condiciones y beneficios con los quetrabajaban, lue go se enfocó en que el colaborador tuviera un desempeño mecánico dirigido a lo administrativo y económico, en cuanto a lo evolución política eindividual las personas iniciaron luchas para garantizar ciertos derechos que eran vulnerados, con el paso del tiempo se ha visto como las organizaciones ven al trabajador como un colaborador con un potencial y talento valioso. Por este motivo es necesario que tenga bienestar laboral, el cual se define como las condiciones de trabajo con el fin de buscar el bienestar y salud mental de la persona.

Para la mayoría de las personas el tener trabajo es esencial en su vida, para su manutención y la de su familia, los trabajadores ocupan como mínimo jornadas laborales al rededor de 8 a 10 horas diarias, sin contar con el desgaste de regreso a casa. Por ello es fundamental que los trabajadores de una empresa se encuentren satisfechos a la hora de realizar sus funciones y obligaciones en su lu- 
gar de trabajo, se sientan cómodos y tengan las condiciones suficientes en su puesto detrabajo, para que no tengan problemas de salud y cumplan con sus funciones correctamente.

\section{El bienestar laboral}

Este ha cambiado a través de la historia, ahora se refiere a las garantías en la estabilidad física, social y familiar del colaborador. Una empresa debe garantizar la carga de míni mos vitales con los que el empleado se sienta seguro y comprometido con su labor. Los Ilamados salarios emocionales son beneficios que pueden influir contundentemente en el momento de que el colaborador tenga que tomar la decisión de ingresar o quedarse dentro de una compañía", dijo Carlos Roldán [20].

Es por esto que incentivar y crear sentido de pertenencia también hacepartedela consolidación del Talento Humano de una organización, ya que de este modo es posi ble conservar aquellas personas que son buenos elementos para la empresa; es decir aquellos colaboradores que se caracterizan por su creatividad, innovación, colaboración y proactividad, en este grupo también podemos incluir aquellos que han hecho carrera dentro de la compañía y que en el transcurso de su experiencia han adquirido conocimientos de diferentes procesos por tanto estarán en la capaci dad decompartir sus conocimientos con nuevos colaboradores y difundir las políticas corporativas.

Lo que se desea con el Bienestar Laboral es el desarrollo eimplementación de programas y actividades que promuevan en los empleados: seguridad (vivienda, beneficios, auxilios, estabilidad), sostenimiento (salud, recreación, esparcimiento) y desarrollo (educación, capacitación); dependiendo el tamaño de la empresa, el Bienestar Laboral de dará desde éste departamento o desde el área de Talento Humano y/ o Gestión Humana; lo importante es mantener motivados a los trabajadores puesto que ello hace que trabajen por y para la empresa, desde el punto de vista Directivo se evidencia por parte de los trabajadores el incremento del sentido de pertenencia, gratitud, lealtad, satisfacción, honorabilidad y compromiso.

La verdadera razón de ser del Bienestar Laboral es que la motivación del personal desencadene en productividad por tanto también adicional a las actividades y programas en los que pueda participar $\mathrm{e}$ trabajador y su familia es indispensablepromover el buen trato, acompañamiento en los procesosy ofrecer un sano ambiente y entorno laboral, además de esto con el fin de que el mejoramiento continuo sea una constantees indispensable realizar evaluación de desempeño, seguimiento y planes de mejoramiento que respalden y garanticen la calidad del producto y/ o servicio que ofrecela compañía [21].

Crear un sistema de bienestar laboral y desarrollo organizacional permitirá no solo tener trabajadores felicessino resultados más eficientes para la organización [22].

\section{Conclusiones}

En la investigación realizada se evidenciaron aspectos en organizaciones colombianas, queno son tomadas en cuenta al momento de evaluar el comportamiento y productividad de sus colaboradores frentea su trabajo y vida familiar. En Colombia se cuenta con profesionales capacitados para la solución de problemas, pero el efecto que crea las organizaciones frente al bienestar laboral no es el adecuado para la motivación al momento de ejercer sus funciones y hace que se sientan poco motivados eimproductivos.

Para ello es necesario la creación de una estrategia competitiva sostenida, que esté soportada y enfocada en el talento humano de la organización con características particulares como habilidades, destrezas y competencias que les permita generar esa ventaja frente a otros, para asumir tales retos es necesario también quelas empresas cuenten con líderes y profesionales altamentecapacitados para proyectarse en la ejecución deactividades de bienestar, que permitan un avance en la estructura interna a partir de una gestión del cambio eficiente con el menor impacto negativo posible en el capital humano queconforma la empresa.

Esta investigación se despliega desde la importancia del desarrollo organizacional y el talento humano, destinando a la empresa a nivel de producción, administración y tecnología la mejora de condiciones a los trabajadores para que de esta manera se reflejeel crecimiento tanto dela empresa como el del colaborador. Las empresas deben asumir este reto en miras de crecer integralmente, generando responsabilidad social y oportunidades 
decrecimiento personal como profesional para sus trabajadores y tener en cuenta quelos seres humanos son lo más importante para la evolución interna y externa de una compañía.

Es por esto que se recomienda la implementación en las empresas, la certificación EFR (Empresa Familiarmente Responsable) la cual se encuentra de la mano con la norma ISO 26000 del 2010. Dicha certificación permite beneficio al empleador - empleado, basándose en el bienestar laboral favoreciendo el entorno socio- económico de la familia y empresa, para mejorar la estabilidad laboral y el compromiso frente a la labor a desempeñar del colaborador.

En Colombia hay aproximadamente 53 organizaciones certificadas con este modelo, lo que la convierte en el segundo país a nivel mundial de trás de España según la fundación Masfamilias (www.masfamilias.org).

Laimplementación de estemodelo en más organizaciones en Colombia permitiría un mayor crecimiento y una mejor gestión delas personas quecon su talento, su compromiso, su conocimiento y su dedicación hacen que la empresa pueda dar una mirada a la calidad en el empleo, la flexibilidad temporal y especial, el apoyo a la familia, el desarrollo personal, profesional y la igualdad de oportunidades, para así conseguir que el colaborador realice su labor con el mayor esfuerzo y pasión, además de aumentar su compromiso con la empresa.

\section{Referencias}

[1] M. Linares, «SCIELO,» Martin Linares, Xiomara; Segredo Perez, Alina María y Perdomo Victoria, Irene, 07 11. Disponible en: http:/ / scielo.sld.cu/ scielo.php?script=sci_arttext\&pid $=\$ 0864-2141201$ 3000300014\&lng=esष्षtlng=es. 2013.

[2] W. Deming, «Elsevier» Disponible en: https:/ / www.sciencedirect.com/ science/ article/ pii/ S2215910X14700217\#bib0005. 1982.

[3] W.Deming, «Scielo»Instituteof Techology Center, p. 15, 1982.

[4] Desarrollo Organizacional A mbiental. http:/ / equipo31d11.blogspot.com/ 2010/ 06/ escuela-deldesarrollo-organizacional_20.html

[5] «TheWealth of N ations» A lianza Editorial, pp. 97$102,1776$.
[6] R. Monroy, «Productividad y Factores Humanos» Interciencia, pp. 121-127, 2012.

[7] J. E. Medina, Modelo Integral de Productividad, Bogota: Universidad Sergio A rboleda, 2007.

[8] C. Lauzan, «Formacion PostgradudadeDirectivos» ENSAP, 2010.

[9] Gómez, M. (2015). El aprendizajeorganizacional y su influencia en la innovación dentro dela organización. Revista delngeniería, Matemáticas y Ciencias de la Información, 2(4)

[10] G. C. Hernandez, «Unal» Disponibleen: https:/ / repositorio.unal.edu.co/ bitstream/ handle/ unal/ 12085/ 01242075.1999.pdf?sequence=1\&isA Ilo wed=y. 1999.

[11] M. Gibson, «El comportamiento humano». 2015.

[12] A. M. Segredo, «Elsevier» Disponibleen: https:/ / www.sciencedirect.com/ science/ article/ pii/ S1575181315000212\#bib0150. 2015.

[13] T. Karp, Trasforming Organisations for Organic Growth, EEUU: Routledge, 2006.

[14] G. S. A mbriz, «Sistema del nformacion Cientifica» Disponible en: https:/ / www.redalyc.org/ pdf/ 635/ 63511932013.pdf. 2009.

[15] J. C. Castaño, «Red Revista Cientificas de A merica» Disponibleen: https:/ / www.researchgate. net/ publication/26544207_Estrategia_orga nizacional_orientada_al_logro_de_resultādos_ desde_el_trabajo_en_équīpo. $20 \overline{0} 7$.

[16] M. T. Rodriguez, «Scielo» Disponible en: http:/ / www.scielo.org.co/scielo.php?script=sci_arttext \&pid=S0120-81602012000100004\&lang=es. 2012.

[17] J. M. Gonzalez, «ResearchGate» Disponible en: https:/ / www.researchgate.net/ profile/ Jose Gonzalez-Paramo/ publication/ 321965972_Cuarta_Revolucion_Industrial_empleo_y_Estad̄o_del Bienestar/ links/ 5a3b89d70f7e9bbef9fece79/ Cuarta-Revolucion-Industrial-empleo-y-Estadodel-Bienestar.pdf. 2017.

[18] W. H. Organizatio, «Who» Disponibleen: https:/ / www.who.int/ occupational_health/ evelyn hwp_spanish.pdf. 2010.

[19] G. N ormativo, «Funcion Republica» Disponibleen: https:/ / www.funcionpublica.gov.co/ eva/ gestor normativo/ norma.php?i=281. 1990.

[20] Chiavenato, «A cademia» Disponibleen: https:/ / www.academia.edu/ 35952063/ CHIAVENATO Idal berto._Gesti\%C3\%B3n_del_talento_humano._ 3ra_Edici\%̄C3\%B3n._McGraw_Hill. $2 \overline{0} 02$.

[21] Y. E. Rodriguez, «TUR Y DES» Disponibleen: http:/ / www.eumed.net/ rev/ turydes/ 08/ errr. htm. 2010.

[22] H. P. Hafemann, «Desarrollo Organizacional,» Ventajas y Procedimientos, 0109 2017. [En línea]. A vailable: http:/ / www.relaciones-humanas.net/ desarrolloorganizacional.html. [Último acceso: 14 03 2020].

Rev. Ingeniería, M atemáticas y Ciencias de la Información Vol. 7 / N úm. 14 / julio - diciembre de 2020; pág. 117-126 